\title{
RNF213 Polymorphism in Intracranial Artery Dissection
}

\author{
Jong S. Kim, Han Bin Lee, Hyuck Sung Kwon \\ Department of Neurology, Asan Medical Center, University of Ulsan College of Medicine, Seoul, Korea
}

\section{Dear Sir:}

Cervicocerebral artery dissections (CADs) account for approximately 20\% of strokes in young individuals. ${ }^{1}$ CADs are associated with trauma or neck rotation, but such history is absent in many patients. One postulated cause of CAD is an underlying arteriopathy, ${ }^{1}$ possibly associated with genetic predisposition. There appears to be an ethnic difference in the location of CAD; in non-Asians, intracranial dissections (IC-CADs) account for $<10 \%$ of all CADs, ${ }^{2}$ whereas in Asians, IC-CAD is more common than extracranial artery dissections (EC-CADs). ${ }^{3,4}$ Genetic variation may explain these differences.

The ring finger protein 213 (RNF213) gene, in the 17q25-ter region, is one of the genes shown to be associated with the ethnic difference in the location of cerebral vascular diseases. RNF213 was initially identified as a susceptibility gene for moyamoya disease, ${ }^{5}$ but RNF213 variants are also associated with non-moyamoya vascular diseases. ${ }^{6}$ Although there are some exceptions, ${ }^{7}$ this genetic variant is mostly associated with intracranial arterial diseases, such as intracranial atherosclerosis. Thus, we hypothesized that RNF213 polymorphisms may be associated with IC-CAD, and assessed this gene in IC-CAD patients compared with age-sex matched normal subjects, as well as with EC-CAD patients.

We prospectively and consecutively enrolled patients diagnosed with IC-CAD or proximal internal carotid artery (ICA) dissection, as confirmed by appropriate imaging techniques, and who were admitted to Asan Medical Center from March 2014 to February 2017. Magnetic resonance angiography (MRA) or computed tomographic angiography (CTA) was initially used for diagnosis. Duplex sonography, high-resolution magnetic resonance imaging, or digital subtraction angiography (DSA) was also performed when needed. Imaging diagnosis of CAD was made when there were: double lumen (a false lumen or an intimal flap), an intramural hematoma, a nonatherosclerotic tapered, flame-shaped stenosis/occlusion or pearland-string sign, or a dissecting aneurysm at a non-branching site. $^{3}$ Age- and sex-matched controls, who visited due to a non-stroke diagnosis, such as tension-type headache and peripheral vestibulopathy, were enrolled from an outpatient clinic.

To assess RNF213 polymorphisms, genomic DNA was extracted from peripheral blood using a Puregene Blood Kit (Qiagen, Hilden, Germany). To identify the major single nucleotide polymorphisms of RNF213 in East Asian patients ${ }^{8}$ (i.e., p.D4013N, p.P4608S, p.R4810K, p.R4853K, p.D4836N, and p.E4950D), three exons (exons 44, 60, and 62) and the appropriate exon-intron boundaries of RNF213 were amplified by polymerase chain reaction and directly sequenced using an ABI3130x/ Genetic Analyzer (Applied Biosystems, Foster City, CA, USA), according to manufacturer instructions. The results were compared with established human RNF213 sequences (GenBank accession no. NM_001256071.1).

All statistical analyses were performed using SPSS version 21.0 software (IBM Co., Armonk, NY, USA). Categorical variables were compared using the chi-square test or Fisher's exact test, and continuous variables were compared using a MannWhitney U test. A P-value $<0.05$ was considered statistically significant. This study was approved by the local Institutional Review Board, and informed consent was obtained from all participants.

We enrolled 24 patients with IC-CAD (21 middle cerebral artery, two intracranial ICA, and one posterior cerebral artery). 
Table 1. Baseline characteristics and prevalence of the RNF213 variant

\begin{tabular}{lccc}
\hline Characteristic & $\begin{array}{c}\text { Control } \\
(\mathrm{n}=24)\end{array}$ & $\begin{array}{c}\text { Subjects } \\
(\mathrm{n}=24)\end{array}$ & $P$ \\
\hline Demographics & & & \\
Age (yr) & $42.9 \pm 8.4$ & $41.8 \pm 10.2$ & $0.657^{*}$ \\
Male sex & $7(29.2)$ & $8(33.3)$ & 1.000 \\
Medical history & & & \\
Hypertension & $1(4.2)$ & $8(25.0)$ & 0.063 \\
Diabetes mellitus & $0(0)$ & $3(9.4)$ & 0.252 \\
Hyperlipidemia & $4(16.7)$ & $6(25.0)$ & 0.724 \\
Smoking & $4(16.7)$ & $6(25.0)$ & 0.724 \\
Coronary artery disease & $0(0)$ & $0(0)$ & $\mathrm{NC}$ \\
Family history of moyamoya disease & $0(0)$ & $1(4.2)$ & 1.000 \\
RNF213 variant & $1(4.2)$ & $8(33.3)$ & 0.023 \\
\hline
\end{tabular}

Values are presented as mean \pm standard deviation or number (\%). Fisher's exact test.

RNF213, ring finger protein 213; NC, not calculated.

*Mann-Whitney $\mathrm{U}$ test were used.

Eleven had ischemic stroke, seven had transient ischemic attack, and three had headache. Three patients were asymptomatic and were admitted to evaluate the cause of intracranial artery stenosis. Of the 24 patients, only two patients had a history of head or neck trauma, including excessive neck rotation. The diagnostic imaging techniques used were MRA $(n=21)$, CTA $(n=3), D S A(n=13)$, and high-resolution MRA (HRMRA; $n=21)$. Twenty-four age- and sex-matched subjects and eight extracranial ICA dissection patients were used as controls. For the eight patients with EC-CAD, a history of trauma was obtained in two patients, and the diagnostic imaging techniques used were MRA $(n=7), \operatorname{CTA}(n=2), \operatorname{DSA}(n=6), \operatorname{HRMRA}(n=3)$, and duplex sonography $(n=3)$.

Baseline characteristics were similar between IC-CAD patients and controls, although dissection patients tended to have hypertension more often. Of the 24 patients with IC-CAD, eight (33.3\%) had an RNF213 variant. Among various single nucleotide polymorphisms of RNF213 gene, only the heterozygote of the p.R4810K (c.14576G $>A$ ) variant was found in our patients, the prevalence of which was significantly higher $(P=0.023)$ in IC-CAD patients than in controls (Table 1). When adjusted for hypertension and RNF213 polymorphism, both hypertension (adjusted odds ratio [OR], 10.185; 95\% confidence interval [Cl], 1.066 to $97.305 ; P=0.04)$ and the presence of the RNF213 variant (adjusted OR, 14.247; 95\% Cl, 1.563 to 129.841; $P=0.018$ ) were independently associated with IC-CAD. However, none of the eight extracranial ICA dissection patients had RNF213 variants. One IC-CAD patient with the RNF213 variant had a family history of moyamoya disease.
We found that one-third of IC-CAD patients had the RNF213 variant, suggesting that this variant may be associated with IC-CAD. As none of the EC-CAD patients had this genetic variant, RNF213 alleles may not be causally associated with the dissection pathology per se, but may increase the vulnerability of the intracranial artery for developing dissection. Interestingly, among the patients with IC-CAD, one had a family history of moyamoya disease. It has been suggested that RNF213 polymorphism is a non-specific marker that increases vulnerability for intracranial arterial disease, and secondary insults or other genetic factors may determine the ultimate phenotype in these patients. ${ }^{6}$ As RNF213 variants are more prevalent in East Asians than in Caucasians, ${ }^{5}$ this genetic difference may explain the higher risk of intracranial arterial diseases, including IC-CAD, in Asia.. Our study is limited due to the small number of enrolled patients. Further studies are needed to confirm our preliminary findings.

\section{References}

1. Schievink WI. Spontaneous dissection of the carotid and vertebral arteries. N Engl J Med 2001;344:898-906.

2. Guillon $B$, Lévy $C$, Bousser MG. Internal carotid artery dissection: an update. J Neuro/ Sci 1998;153:146-158.

3. Kwon JY, Kim NY, Suh DC, Kang DW, Kwon SU, Kim JS. Intracranial and extracranial arterial dissection presenting with ischemic stroke: Lesion location and stroke mechanism. J Neurol Sci 2015;358:371-376.

4. Kim BM, Kim SH, Kim DI, Shin YS, Suh SH, Kim DJ, et al. Outcomes and prognostic factors of intracranial unruptured vertebrobasilar artery dissection. Neurology 2011;76:1735-1741.

5. Fujimura $M$, Sonobe $S$, Nishijima $Y$, Niizuma $K$, Sakata $H$, Kure $S$, et al. Genetics and biomarkers of moyamoya disease: significance of RNF213 as a susceptibility gene. J Stroke 2014;16:65-72.

6. Bang OY, Fujimura M, Kim SK. The pathophysiology of moyamoya disease: an update. J Stroke 2016;18:12-20.

7. Fukushima H, Takenouchi T, Kosaki K. Homozygosity for moyamoya disease risk allele leads to moyamoya disease with extracranial systemic and pulmonary vasculopathy. Am J Med Genet A 2016;170:2453-2456.

8. Liu W, Morito D, Takashima S, Mineharu Y, Kobayashi H, Hitomi $T$, et al. Identification of rnf213 as a susceptibility gene for moyamoya disease and its possible role in vascular development. PLoS One 2011;6:e22542.

9. Yang WJ, Wong KS, Chen XY. Intracranial atherosclerosis: from microscopy to high-resolution magnetic resonance imaging. J Stroke 2017;19:249-260. 
Correspondence: Jong S. Kim

Department of Neurology, Asan Medical Center, University of Ulsan College of

Medicine, 88 Olympic-ro 43-gil, Songpa-gu, Seoul 05505, Korea

Tel: +82-2-3010-3442

Fax: +82-2-474-4691

E-mail: jongskim@amc.seoul.kr

Received: July 24, 2018

Revised: August 31, 2018

Accepted: September 1, 2018

This work was supported by a grant from the Ministry for Health, Welfare, and

Family Affairs, Republic of Korea (HI14C1985).

The authors have no financial conflicts of interest. 